\title{
Editorial
}

\section{MIMO Antenna Design and Channel Modeling 2013}

\author{
Wenhua Chen, ${ }^{1}$ Manos M. Tentzeris, ${ }^{2}$ Yuan Yao, ${ }^{3}$ Yan Zhang, ${ }^{4}$ and Li Yang \\ ${ }^{1}$ Department of Electronic Engineering, Tsinghua University, Beijing 100084, China \\ ${ }^{2}$ The School of Electrical and Computer Engineering, Georgia Institute of Technology, Atlanta, GA 30332-0250, USA \\ ${ }^{3}$ School of Electronic Engineering, Beijing University of Posts and Telecommunications, Beijing 100876, China \\ ${ }^{4}$ Beijing Institute of Technology, Beijing 100081, China \\ ${ }^{5}$ Texas Instruments, Dallas, TX 75266-0199, USA \\ Correspondence should be addressed to Wenhua Chen; chenwh@tsinghua.edu.cn
}

Received 30 September 2013; Accepted 30 September 2013

Copyright (C) 2013 Wenhua Chen et al. This is an open access article distributed under the Creative Commons Attribution License, which permits unrestricted use, distribution, and reproduction in any medium, provided the original work is properly cited.

With the fast evolution of wireless communication standards such as LTE-A, the fifth generation (5G) mobile and wireless communication technologies are emerging into research fields. Based on the Internet Protocol architecture of $4 \mathrm{G}$ communication systems, unprecedented numbers of smart and heterogeneous wireless devices will be accessing future $5 \mathrm{G}$ mobile and wireless communication systems with a continuing growth of Internet traffic. Therefore, compared with $4 \mathrm{G}$ communication systems, significantly higher wireless transmission rates are expected in $5 \mathrm{G}$ communication systems, such as $10 \mathrm{Gbps}$ peak data rates with $8-10 \mathrm{bps} / \mathrm{Hz} /$ cell. Moreover, energy efficiency concepts will be fully integrated into future wireless communication systems to protect the environment. To meet the above challenges, $5 \mathrm{G}$ mobile and wireless communication systems will require a mix of new system concepts to boost spectral efficiency, energy efficiency, and the network design, such as massive MIMO technologies, green communications, cooperative communications, and heterogeneous wireless networks. From this perspective, MIMO technology will continue to play an important role in the upcoming $5 \mathrm{G}$ systems.

Electromagnetic (EM) fields can carry not only energy but also angular momentum. The angular momentum is composed of spin angular momentum (SAM) and orbital angular momentum (OAM) describing its polarization state and the phase structure distribution, respectively. Recently, more and more attention has been paid to OAM in both optical and radio domain. It is worthy to be mentioned that Edfors et al. compared the technique of using OAM states of radio waves with traditional MIMO communication methods. Simulations showed that, for certain array configurations in free space, traditional MIMO theory leads to eigenmodes identical to the OAM states. According to this result, they concluded that communicating over the subchannels given by OAM states is a subset of the solutions offered by MIMO. In the foreseeable future, the discussion about the OAM and MIMO will be the key focus areas.

In this special issue, we have received 17 paper submissions and accepted 11 ones finally according to the reviewers' comment and associate editors' suggestion. These accepted papers include MIMO antenna design, MIMO channel modeling, massive MIMO, and OAM technologies.

Dr. B. Mun from Kwangwoon University of Korea proposed a compact handset multiple-input-multiple-output (MIMO) antenna for Long Term Evolution (LTE) 700 band (746-787 MHz) applications. Dr. W.-Q. Wang from UESTC of China designed the MIMO antenna array with the polynomial factorization method.

Dr. P. Handayani from Institut Teknologi Sepuluh Nopember of Indonesia proposed a model of doubledirectional indoor non-line-of-sight (NLOS) channels for multiterminal communications and completed their model by characterizing the parameters of double-directional channel impulse response of such channels through measurements in indoor environment using 3D synthetic array antenna at $2.5 \mathrm{GHz}$ band. Dr. Z. Mansor from Bristol University of United Kingdom analyzed the impact of 3D multipath in an LTE-advanced heterogeneous network. 
Dr. Deng from Tsinghua University of China gave a feasible and simple solution of generating OAM-carrying radio beams and proposed an OAM antenna system which is consisted of 8-Vivaldi connected antenna elements sequentially and folded into a hollow cylinder.

We sincerely hope that this special issue can further help the readers to understand MIMO design and MIMO channel modeling and explore the future 5G MIMO in the subsequent development of the system.

Wenhua Chen Manos M. Tentzeris Yuan Yao

Yan Zhang Li Yang 

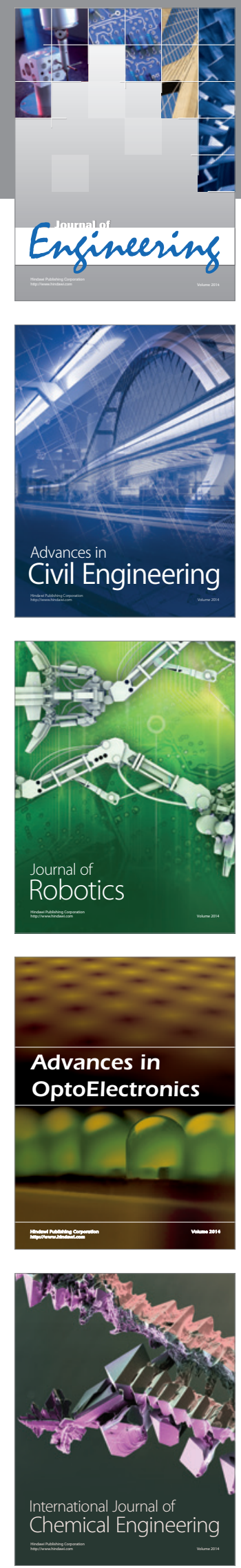

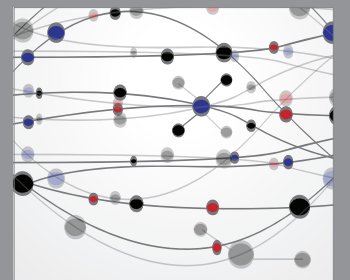

The Scientific World Journal
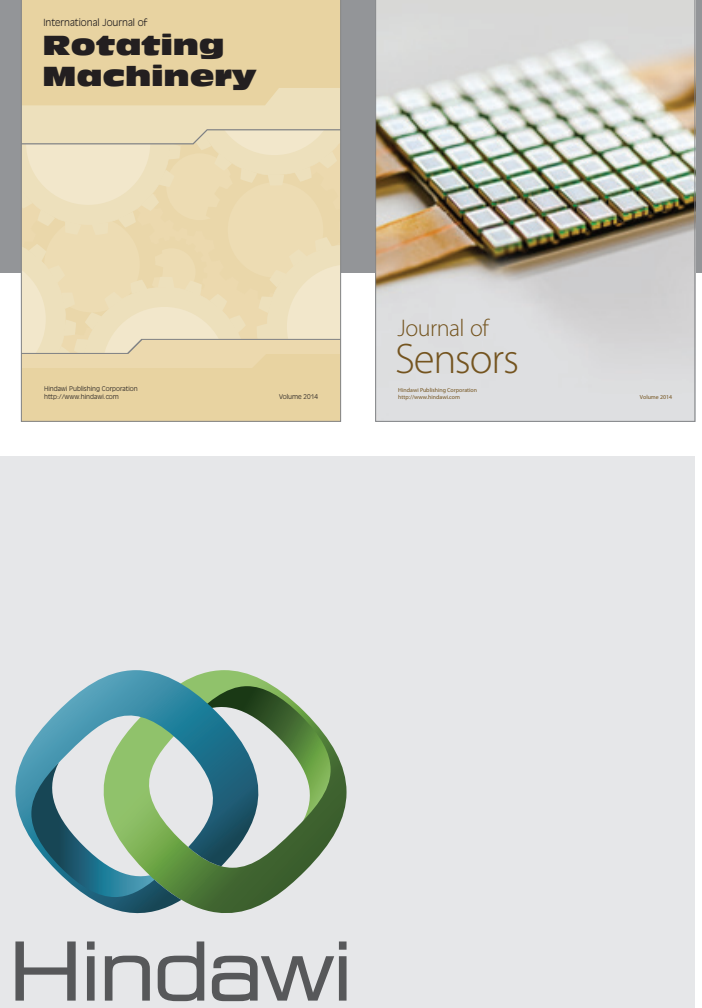

Submit your manuscripts at http://www.hindawi.com
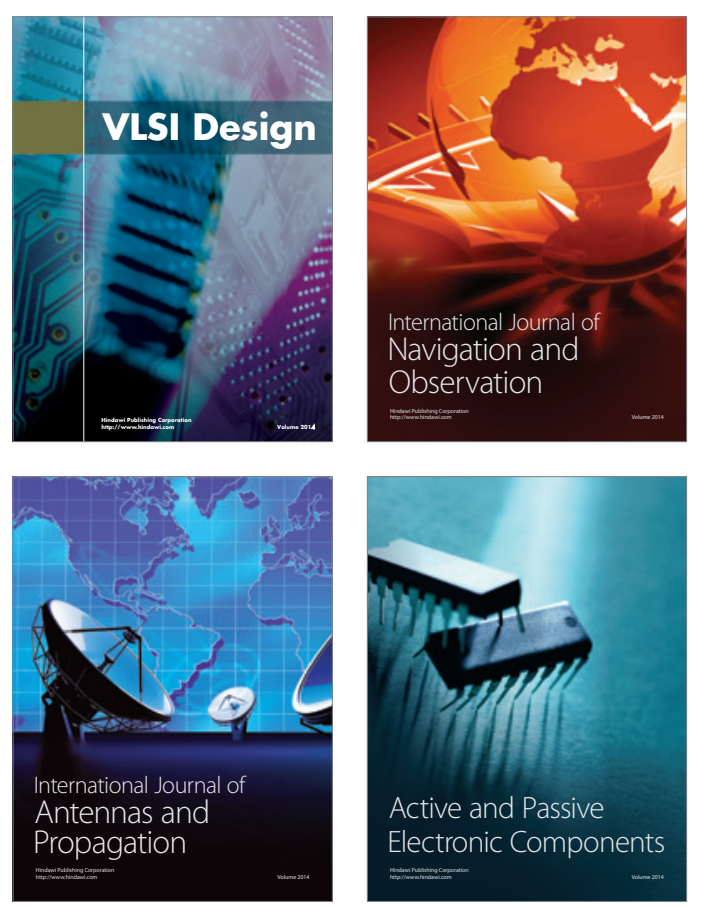
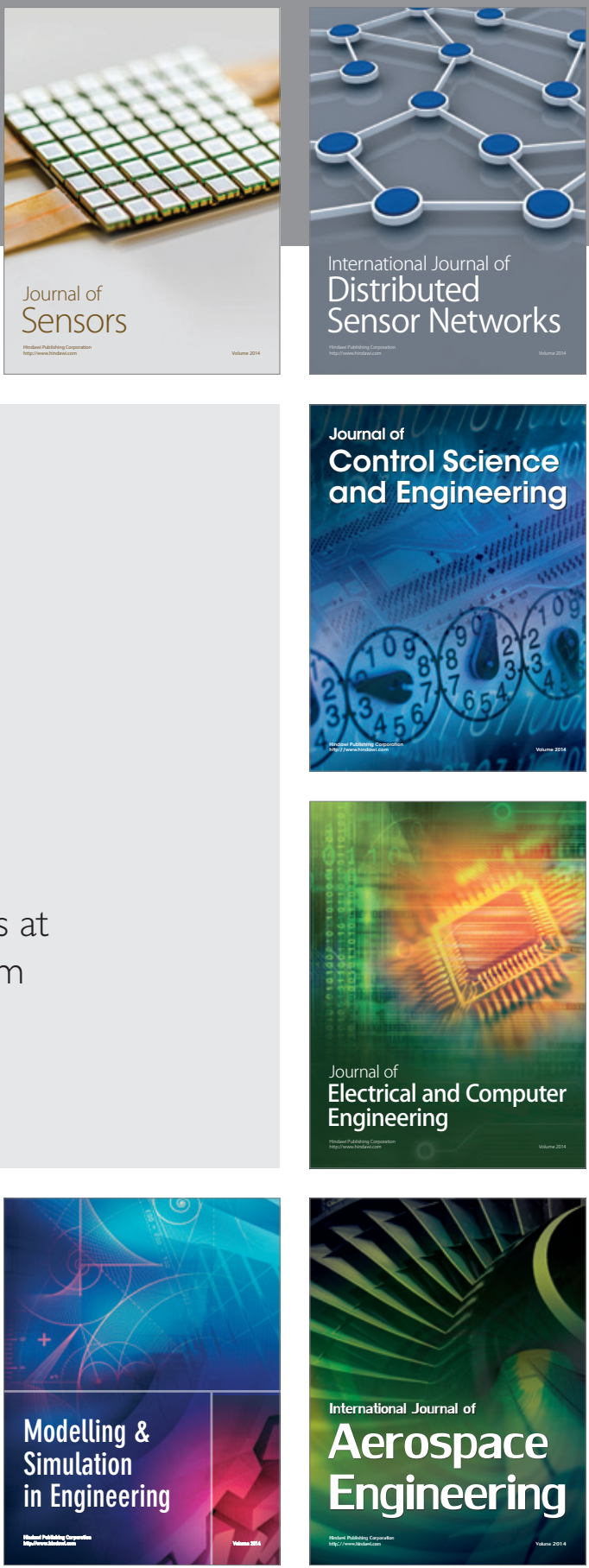

Journal of

Control Science

and Engineering
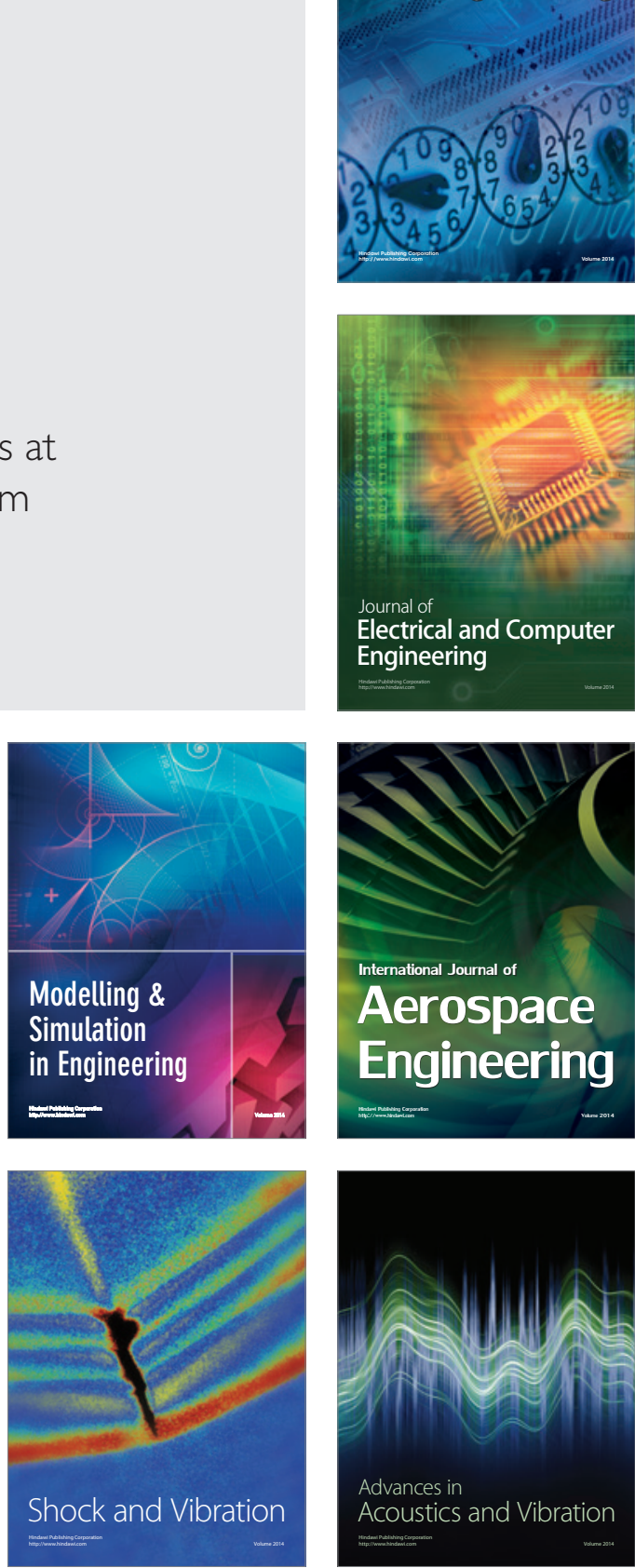